Pacific Journal of Mathematics

TRANSITIVE ISOMETRY GROUPS WITH NONCOMPACT ISABEL DotTy D 


\section{TRANSITIVE ISOMETRY GROUPS WITH NON-COMPACT ISOTROPY}

\section{Dotti Miatello and R. J. Miatello}

Let $G$ be a connected Lie group acting effectively and transitively by isometries on a riemannian manifold $M$. Then $G$ is a Lie subgroup of the full isometry group, which is not necessarily closed. In this paper we study the structure of the closure of $G$ in $I(M)$ and illustrate the results with examples, with non-compact isotropy, where the closure is described explicitly.

Introduction. If $M$ is a riemannian manifold the isotropy group in $I(M)$ is compact; hence a homogeneous riemannian $M$ can always be represented as a quotient $G^{\prime} / H^{\prime}$ with $H^{\prime}$ compact.

Assume now that $G$ is a connected Lie group acting effectively and transitively by isometries on $M$. Then $G$ is a Lie subgroup of $I(M)$ which will be closed in $I(M)$ if and only if the isotropy subgroup $H$ is compact.

In this paper we study in detail the closure of $G$ in $I(M)$. Also if $G$ is any connected Lie group and $H$ a closed subgroup we compare three standard conditions on $H$ which ensure that $G / H$ admits a riemannian invariant structure. The rest of the paper is devoted to illustrate the fact that it is rather common to have transitive, effective, non-closed Lie subgroups of $I(M)$, hence the isotropy subgroup is non-compact. This situation arises quite frequently, even when $M$ is compact (Lemma 1.4). Also, any connected semisimple Lie group with infinite center admits a closed non-compact subgroup $H$ such that $G$ acts effectively on $G / H$ and $G / H$ carries a $G$-invariant riemannian structure. In this case, that is, when $G$ is semisimple, we give an upper bound for the dimension of $\bar{G}_{L}$ and provide examples showing that these bounds are sharp (see (2.4), Proposition 2.3 and Remark 2.4).

In [DMW] the use of $\bar{G}_{L}$ proved to be convenient in the study of bounded isometries on a riemannian manifold acted on transitively and effectively by a semisimple Lie group without local compact factors. Also, some examples where $G_{L}$ is not closed in $I(M)$ ( $G$ semisimple) are given in [DMW] Example (3.10). The authors would like to thank J. A. Wolf for very useful comments on a first version of this paper and, in particular, for suggesting a simpler proof of Proposition 2.2. 
1. Closure of $G$. Let $G$ be a connected Lie group with Lie algebra $g$ and let $H$ be a closed subgroup of $G$ with Lie algebra $\mathfrak{h}$. We will say that $H$ satisfies condition (1) (respectively (2), (3)) if

(1) $\overline{\operatorname{Ad}_{G}(H)}$ is compact in $\mathrm{GL}(g)$, the closure with respect to the $\mathrm{GL}(\mathrm{g})$ topology;

(2) $\operatorname{cl}\left(\operatorname{Ad}_{G}(H)\right)$ is compact in $\operatorname{Ad}(G)$, where cl denotes closure with respect to the $\operatorname{Ad}(G)$ topology;

(3) $\mathfrak{h}$ is a compactly embedded subalgebra of $\mathfrak{g}$ (or equivalently see [H, p. 130]) $\operatorname{Ad}_{G}\left(H_{0}\right)$ is compact in $\left.\operatorname{Ad}(G)\right)$, where $H_{0}$ denotes the identity component of $H$.

Along this section we will analyze the relationship among the different conditions (1), (2), (3).

Condition (1). It is well known (cf. [Ch E]. Ch. III) that if $G$ acts effectively on $G / H$ then $H$ satisfies (1) if and only if $G / H$ admits a $G$-invariant riemannian structure. Furthermore for each such structure, $G$ is a Lie subgroup of the full isometry group $I(M), M=G / H$, via $x \rightarrow L_{x}, L_{x}$ the left translation on $M=G / H$. If $S$ is a Lie subgroup of $G$, denote $S_{L}=\left\{L_{x} \mid x \in S\right\}$. The next theorem describes the closure of $G_{L}$ in $I(M)$.

1.1. TheOREM. Let $G$ be a connected Lie group acting effectively on $M=G / H, H$ a subgroup of $G$ satisfying (1). Then

$$
\bar{G}_{L}=\left\{L_{x} \circ \varphi: x \in G, \varphi \in \bar{H}_{L}\right\} .
$$

In particular, $G_{L}$ is closed if and only if $H$ is compact. Moreover

(i) $H_{L}$ is isomorphic to $\overline{\mathrm{Ad}(H)}$ (as Lie groups).

(ii) There is an exact sequence

$$
\{1\} \rightarrow\left\{\left(x, \operatorname{Ad}\left(x^{-1}\right)\right): x \in H\right\} \rightarrow G \rtimes \overline{\operatorname{Ad}(H)} \rightarrow \bar{G}_{L} \rightarrow\{1\}
$$

where $\rtimes$ denotes semidirect product.

Proof. If $L_{x_{n}}$ converges in $I(M)$ then $x_{n} H \rightarrow x^{*} H$ in $G / H$, for some $x^{*}$ in $G$. Hence there is a sequence $h_{n}$ in $H$ such that $x_{n} h_{n} \rightarrow x^{*}$ in $G$. Now $L_{h_{n}^{-1}} \in H_{L}$ and since $H_{L}$ is contained in the isotropy subgroup in $I(M), \quad L_{h_{n}^{-1}} \rightarrow \phi, \phi \in \bar{H}_{L}$. As a consequence $L_{x_{n_{j}}}=L_{x_{n_{j}} h_{n_{j}}} \circ L_{h_{n_{j}}^{-1}}$ converges to $L_{x^{*}}^{\prime} \circ \phi$ and the first assertion follows.

Clearly, if $H$ is compact, $\bar{H}_{L}=H_{L} \subset G_{L}$. Conversely if $G_{L}$ is closed in $I(M)$ then $H_{L}$ is compact in $G_{L}$ since $H_{L}$ is the intersection of $G_{L}$ with the isotropy subgroup in $I(M)$. 
Next we prove (i). Let $\mathfrak{m}$ be an $\overline{\operatorname{Ad}(H)}$ invariant complement of $\mathfrak{h}$ in $\mathfrak{g}$ where $\mathfrak{h}$ denotes the Lie algebra of $H$. It is not hard to show that the canonical map $\bar{H}_{L} \rightarrow \overline{\operatorname{Ad}(H) \mid \mathfrak{m}}$ sending $\varphi \rightarrow(d \varphi)_{e H}$ is an isomorphism of Lie groups. Hence (i) will follow once we prove that $\overline{\operatorname{Ad}(H)}$ and $\overline{\operatorname{Ad}(H) \mid \mathrm{m}}$ are isomorphic.

Let $\pi: \overline{\operatorname{Ad}(H)} \rightarrow \overline{\operatorname{Ad}(H) \mid \mathfrak{m}}$ denote the restriction homomorphism. By compactness of $\overline{\operatorname{Ad}(H)}$ it follows that $\pi$ is onto. If $T$ is in $\overline{\operatorname{Ad}(\underline{H)}}$ and $T \mid \mathfrak{m}$ is the identity, then $T \mid \mathfrak{m}_{1}$ is the identity for any $\mathfrak{m}_{1}$, an $\overline{\operatorname{Ad}(H)}$ invariant complement of $\mathfrak{h}$. Hence we may take $\mathfrak{m}_{1}$ to be the orthogonal complement of $\mathfrak{h}$ with respect to an $\overline{\operatorname{Ad}(H)}$ invariant inner product and consequently $\mathfrak{m}_{1}$ will satisfy $\left[\mathfrak{h}, \mathfrak{m}_{1}\right] \subset \mathfrak{m}_{1}$. If $X \in \mathfrak{h}, Y \in \mathfrak{m}_{1}$

$$
[T X-X, Y]=[T X, Y]-[X, Y]=T[X, Y]-[X, Y]=0 .
$$

Hence $\operatorname{Ad}(\exp (T X-X))$ is the identity in $\mathrm{m}_{1}$ or equivalently $\left(d L_{\exp (T X-X)}\right)_{e H}$ is the identity in $G / H$. Since we are assuming that the action of $G$ on $G / H$ is effective it follows that $\exp (T X-X)=e$ for all $X$ in $\mathfrak{h}$, hence $T$ is the identity on $\mathfrak{g}$.

Now (ii) follows immediately from the description of $\bar{G}_{L}$, after observing that the kernel of the Lie group homomorphism $G \rtimes \bar{H}_{L} \rightarrow \bar{G}_{L}$, $(x, \varphi) \rightarrow L_{x} \circ \varphi$ consists of pairs $\left(x, L_{x^{-1}}\right)$ where $x$ is in $H$.

Let $G$ be a connected Lie group and $H$ a subgroup of $G$ satisfying (1). Set

$$
\operatorname{Aut}(G, H)=\left\{\varphi \in \operatorname{Aut}(G): \varphi(H)=H,(d \varphi)_{e} \in \overline{\operatorname{Ad}(H)}\right\} .
$$

If $\varphi \in \operatorname{Aut}(G, H), \bar{\varphi}: G / H \rightarrow G / H$ will denote the induced diffeomorphism, $\bar{\varphi}(x H)=\varphi(x) H$. If $G$ acts effectively on $M=G / H$ then, for any $G$-invariant riemannian structure on $M, \bar{\varphi}$ is an isometry of $M$. Furthermore, if $(d \varphi)_{e}=\lim \operatorname{Ad}\left(h_{n}\right)$, the sequence $L_{h_{n}} \rightarrow \psi, \psi$ in $\bar{H}_{L}$ (by taking a subsequence if necessary), hence $\left(d L_{h_{n}}\right)_{e H} \rightarrow(d \psi)_{e H}$, that is, $\bar{\varphi}=\psi$ since they both fix $e H$.

1.2. Proposition. Assume the hypotheses of Theorem 1.1 hold. Then the correspondence $\varphi \rightarrow \bar{\varphi}, \operatorname{Aut}(G, H) \rightarrow \bar{H}_{L}$ is a group isomorphism.

Proof. It is clearly a homomorphism. To check it is surjective assume $\psi=\lim L_{h_{n}}, h_{n} \in H$. By taking a subsequence, if necessary, we have that $\operatorname{Ad}\left(h_{n}\right) \rightarrow T, T$ an automorphism of $\mathfrak{g}$. If $\phi$ denotes the lifting of $T$ to $\tilde{G}$, $\tilde{G}$ the simply connected covering group of $G$, then $\phi$ is the identity restricted to the center of $\tilde{G}$. In fact, let $\tilde{h}_{n}$ in $\tilde{G}$ be such that $d\left(I_{\tilde{h}_{n}}\right)_{e}=$ $\operatorname{Ad}\left(h_{n}\right)$. Then $I_{\tilde{h}_{n}}(\exp X)=\exp \operatorname{Ad}\left(h_{n}\right) X \rightarrow \exp T X=\phi(\exp X)$ and since 
$\tilde{G}$ is connected $I_{\tilde{h}_{n}}$ converges pointwise to $\phi$; the assertion follows. As a consequence $\phi$ induces an automorphism $\varphi: G \rightarrow G$ lifting $T$ and since $I_{h_{n}}$ converges pointwise to $\varphi$ then $\varphi(H) \subset H$. By repeating the above argument with $T^{-1}$ we conclude that $\varphi \in \operatorname{Aut}(G, H)$. Also from $I_{h_{n}}(x)$ $\rightarrow \varphi(x), x$ in $G$, it follows that $L_{h_{n}}(x H) \rightarrow \bar{\varphi}(x H)$ and consequently $\bar{\varphi}=\psi$.

Assume $\bar{\varphi}$ is the identity on $G / H$. Then $y^{-1} \varphi(y) \in H$, for all $y$ in $G$. Hence

$$
\left.\frac{d}{d t}\right|_{t=0} \exp (-t Y) \varphi(\exp t Y)=-Y+(d \varphi)_{e} Y \in \mathfrak{h}
$$

for all $Y$ in $g$. As a consequence $(d \varphi)_{e} \mid \mathfrak{m}$ is the identity, $m$ an $\operatorname{Ad}(H)$ invariant complement of $\mathfrak{h}$. Hence $(d \varphi)_{e}$ is the identity on $\mathfrak{g}$ (see the proof of (i), Theorem 1.1) or equivalently, $\varphi=$ Id.

Condition (2). Let Ad: $G \rightarrow \operatorname{Aut}(g)$ be the adjoint representation, $Z$ the center of $G$, ad: $\mathfrak{g} \rightarrow \operatorname{Der}(\mathfrak{g})$ the derivative of $\operatorname{Ad}$. Since $\operatorname{Ad}(G)$, the connected Lie subgroup of $\operatorname{Aut}(\mathfrak{g})$ with Lie algebra $\operatorname{ad}(\mathfrak{g})$, is isomorphic to $G / Z$, condition (2) is equivalent to

$$
\pi(\overline{Z H}) \text { compact in } G / Z \text {, }
$$

where $\pi: G \rightarrow G / Z$ is the standard projection. Furthermore, since Aut( $g$ ) carries the relative topology of $\mathrm{Gl}(\mathfrak{g})$, Condition (2) implies Condition (1) (the converse does not hold in general, see Example 1.4 below). For subgroups $H$ of $G$ satisfying (2), one can give a simple description of $\bar{G}_{L}$, in terms of $G$ and the closure of $Z H$ in $G$ (see also [DMW], (3.5)).

1.3. Proposition. Let $G$ be a connected Lie group, acting effectively on $M=G / H, H$ satisfying (2). Then $\bar{G}_{L}=\left\{L_{x} \circ R_{y^{-1}}: x \in G, y \in \overline{Z H}\right\}$ and there is an exact sequence

$$
\{1\} \rightarrow S \rightarrow G \times \overline{Z H} \rightarrow \bar{G}_{L} \rightarrow\{1\}
$$

where $S=\{(z, z h) \mid z \in Z, h \in H\}$. Furthermore, if $H$ is not compact, $\overline{Z H} / H$ is an abelian Lie subgroup of positive dimension of $Z\left(\bar{G}_{L}\right)$, the center of $\bar{G}_{L}$.

Proof. We observe first that for any $G$-invariant structure on $M=$ $G / H$, if $y \in \overline{Z H}$ the map $R_{y}$ is in the closure of $G$ in $I(M)$, where $R_{y}(z H)=z y H$. In fact, since $\overline{Z H}$ is contained in $N(H)$, the normalizer of $H, R_{y}$ is a diffeomorphism. Furthermore the condition $y=\lim z_{n} h_{n}$ says 
that $x y H=\lim L_{z_{n}}(x H)$, hence $R_{y}=\lim L_{z_{n}}$ in $I(M)([\mathbf{H}]$, p. 203, Lemma 2.4).

The map $\alpha: G \times \overline{Z H} \rightarrow \bar{G}_{L}, \alpha(x, y)=L_{x} \circ R_{y^{-1}}$ is a Lie group homomorphism. To check that $\alpha$ is surjective it is enough (by Theorem 1.1) to consider $\varphi$ in $\bar{H}_{L}, \varphi=\lim L_{h_{n}}$. Since $H$ satisfies (2'), there exists a subsequence $h_{n_{j}}$ such that $\pi\left(h_{n_{j}}\right) \rightarrow \pi(u), u \in \overline{Z H}$. Hence $z_{n_{j}} h_{n_{j}} \rightarrow u$ for some $z_{n_{j}}$ in $Z^{\prime}$ and $L_{h_{n}}(x H)=h_{n} z_{n} x z_{n}^{-1} h_{n}^{-1} H \rightarrow L_{u} \circ R_{u^{-1}}(x H)$, that is, $\varphi=\alpha(u, u)$. Let $(x, y)$ in $\operatorname{ker} \alpha$. Then $z^{-1} x z y^{-1} \in H$ for all $z$ in $G$. Since $y x^{-1} \in H$ (taking $z=e$ ) it follows that $L_{x} \circ R_{x^{-1}} \in \operatorname{Aut}(G, H)$ induces the identity on $G / H$. By Proposition $1.2, L_{x} \circ R_{x^{-1}}$ is the identity on $G$ and the description of $S=\operatorname{ker} \alpha$ follows.

It is not hard to check that $\overline{Z H} / H$ is an abelian Lie group which is a Lie subgroup of $Z\left(\bar{G}_{L}\right)$. Assume $\operatorname{dim} \overline{Z H} / H=0$. The map

$$
\beta: Z \times H \rightarrow \overline{Z H}, \quad(z, h) \rightarrow z h
$$

is a one-to-one Lie group homomorphism. By regularity $\beta(Z \times H)=Z H$ is open in $\overline{Z H}$, hence closed. Now if $Z H$ is closed and $H$ satisfies $\left(2^{\prime}\right)$ it follows that $H$ is compact. This completes the proof.

We will next show that, even the case $M$ compact riemannian, it is quite common to have a representation with non-compact isotropy subgroup. This question was posed to us by Cristian Sanchez.

1.4. LemMA. Let $G$ be a compact, connected semisimple Lie group, H a closed subgroup of $G$ such that $G$ acts effectively on $G / H$. Assume that $M=G / H$ carries a G-invariant riemannian structure such that $\operatorname{rank}(U) \geq$ $\operatorname{rank}(H)+2$, where $U=\left\{x \in N(H): R_{x}\right.$ is an isometry $\}$. Then $M$ can be represented as a quotient $G^{\prime} / H^{\prime}$, where $G^{\prime}$ acts effectively by isometries on $G^{\prime} / H^{\prime}$ and $H^{\prime}$ is a closed noncompact subgroup. Furthermore, if $\phi$ : $G^{\prime} \rightarrow I(M)$ is the inclusion as a Lie subgroup, then $\phi\left(G^{\prime}\right)=G_{L} \cdot S_{R}$ where $S$ is a torus in $U$.

Proof. Let $\mathfrak{u}$ denote the Lie algebra of $U$ and let $T$ be a torus in $U$ such that $\operatorname{dim}(T) \geq 2$ and $\mathrm{t} \cap \mathfrak{h}=0$ (here $\mathrm{t}$ is the Lie algebra of $T$ ). Fix $X \in \mathrm{t}$ so that $t \rightarrow \exp (t X)$ is one-to-one.

Set $G^{\prime}=G \times \mathbf{R}$; let $\phi: G^{\prime} \rightarrow I(M)$ be given by $\phi(g, t)=$ $L_{g} \circ R_{\exp (-t X)}$. We assert that $\phi$ is a monomorphism. Indeed, $(g, t) \in$ $\operatorname{ker}(\phi)$ if and only if $x^{-1} g x \exp (-t X) \in H$, for any $x$ in $G$. Thus $g=$ $h \exp (t X) \in U$, for some $h$ in $H$ and $\operatorname{Ad}(g)(Z)-Z \in \mathfrak{h}$, for any $Z \in \mathfrak{g}$. Arguing as in the proof of Proposition 1.2 one concludes that $\operatorname{Ad}(g)=\mathrm{Id}$, or $g \in Z(G)$. On the other hand, the one-parameter group generated by $X$ 
intersects $H$ in the identity element, since $T \cap H$ is a finite group. This easily implies that $h=\exp (t X)=e$, or $t=0$. Hence $M$ is isometric to $G^{\prime} / H^{\prime}$ where $H^{\prime}=\{(h \exp (t X), t): h \in H, t \in \mathbf{R}\}$.

Finally, by Proposition 1.3 it is easy to check that if $S$ denotes the closure of $\{\exp (t X): t \in \mathbf{R}\}$, then

$$
\overline{G_{L}^{\prime}}=G_{L} \cdot(S \times\{0\})_{R} .
$$

From this it easily follows that $\overline{\phi\left(G^{\prime}\right)}$ is as asserted in the lemma. This completes the proof.

1.5. Remark. (i) As a particular case in the lemma we may take $H=\{e\}$. That is, $M=G$ is any compact, semisimple, connected Lie group endowed with a left invariant metric such that $\operatorname{rank}(U) \geq 2$. For instance, if $\operatorname{rank}(G) \geq 2$ and we use a bi-invariant metric, then $G$ is a riemannian symmetric space admitting a representation $G^{\prime} / H^{\prime}$, with $H^{\prime}$ non-compact.

(ii) The proof above only uses that $Z(G)$ is finite and that $H$ is compact. Hence the lemma remains valid under these assumptions on $G$ and $H$, only.

We recall that in a variety of cases Conditions (1) and (2) are equivalent. For instance when $\operatorname{Ad}(G)$ is closed in $\operatorname{GL}(g)$ since both topologies coincide. This happens for $G$ semisimple since any derivation of $\mathrm{g}$ is inner. Also $\operatorname{Ad}(G)$ is closed if $G$ is reductive. It may fail to be so when $G$ is solvable as the next example shows.

1.6. EXAMPLE. We provide here an example of a subgroup $H$ of a connected Lie group $G$, satisfying (1) and not satisfying (2). Moreover since $G$ acts effectively on $G / H$, we apply Theorem 1.1 to compute the closure of $G$ in $I(M), M=G / H$.

If $t_{i} \in S^{1}, z_{l} \in \mathbf{C}$ for $1 \leq i \leq n+1$, denote

$$
\begin{aligned}
& s\left(t_{1}, \ldots, t_{n+1}\right)=\left|\begin{array}{llll}
t_{1} & & & \\
& \ddots & & \\
& & t_{n+1} & \\
& & & 1
\end{array}\right|, \\
& n\left(z_{1}, \ldots, z_{n+1}\right)=\left|\begin{array}{cccc}
1 & & & z_{1} \\
& \ddots & & \vdots \\
& & 1 & z_{n+1} \\
& & & 1
\end{array}\right|
\end{aligned}
$$


and set

$$
S=\left\{s\left(t_{1}, \ldots, t_{n+1}\right): t_{i} \in S^{1}\right\}, \quad N=\left\{n\left(z_{1}, \ldots, z_{n+1}\right): z_{i} \in \mathbf{C}^{n+1}\right\},
$$

$G_{1}=N S$. Since

$$
s\left(t_{1}, \ldots, t_{n+1}\right) n\left(z_{1}, \ldots, z_{n+1}\right) s\left(t_{1}, \ldots, t_{n+1}\right)^{-1}=n\left(t_{1} z_{1}, \ldots, t_{n+1} z_{n+1}\right)
$$

then it is clear that $G_{1}$ is a closed subgroup of $\mathrm{GL}(n+2, \mathrm{C})$ isomorphic to the semidirect product $N \rtimes S$ where $S$ acts on $N$ by

$$
s\left(t_{1}, \ldots, t_{n+1}\right) \cdot\left(n\left(z_{1}, \ldots, z_{n+1}\right)\right)=n\left(t_{1} z_{1}, \ldots, t_{n+1} z_{n+1}\right) .
$$

We note that if $g=n s, g$ commutes with $N$ if and only if $s$ commutes with $N$, that is, $s=1$. Hence, if $G_{2}$ is a Lie subgroup with $N \subset G_{2}$ and if, for each $j$, there is $s\left(t_{1}, \ldots, t_{n+1}\right)$ in $S \cap G_{2}$ with $t_{j} \neq 1$, then $Z\left(G_{2}\right)=1$.

Now let

$$
\phi: \mathbf{R}^{n+1} \rightarrow G_{1}, \phi\left(x_{1}, \ldots, x_{n+1}\right)=s\left(e^{2 \pi i x_{1}}, \ldots, e^{2 \pi i x_{n+1}}\right) .
$$

Let $\mathbf{V}$ be an $n$-dimensional subspace of $\mathbf{R}^{n+1}$ and let $v \in \mathbf{V}$ be such that $\phi$ is injective when restricted to $\mathbf{V}$ and $\mathbf{R} v+\sum_{1}^{n+1} \mathbf{Z} e_{j}$ is dense in $\mathbf{R}^{n+1}$, $\left\{e_{1}, \ldots, e_{n+1}\right\}$ the standard basis of $\mathbf{R}^{n+1}$; (see appendix, Lemma A.4).

Set $G=N \phi(\mathbf{V}), H=\phi(\mathbf{R} v)$. Then $G$ is a normal dense Lie subgroup of $G_{1}$ and $H$ is dense in $S$. On the other hand, $H$ is a closed Lie subgroup of $G$. Now, by the choice of $H$, it is not hard to see that $\overline{\operatorname{Ad}(H)}=\operatorname{Ad}_{G}(S)$ and this is, in turn, isomorphic to $T^{n+1}$. Hence (1) holds. However, given that $Z=\{e\}$, and $H$ is not compact, (2) is not satisfied.

One checks that $\overline{\operatorname{Ad}(H)}=\left\{I_{s}: s \in S\right\}$. From this and the fact that $G$ acts effectively on $G / H$, it follows (see 1.1 and 1.2) that

$$
\bar{G}_{L}=\left\{L_{x} \circ \bar{I}_{s}: x \in G, s \in S\right\} .
$$

By a calculation one checks that $Z\left(\bar{G}_{L}\right)=\{e\}$ in contrast to the case when (2) is satisfied (see Proposition 1.3).

1.7. Remark. Clearly a connected subgroup $H$ of $G$ satisfying condition (3) will satisfy (2) (then (1)). Now if $G$ acts effectively on $G / H$, the conditions $\operatorname{Ad}_{G}(H)$ compact and $H$ compact are equivalent. Hence if $H$ satisfies (3), $G_{L}$ will be closed in $I(M)$. As $1.4,1.5$, and 1.6 show, it is not true in general that if $G / H$ is riemannian homogeneous then $H$ satisfies (3). On the other hand, we see later (see Remark 2.3) that if $G$ is any simply connected semisimple Lie group with infinite center there exists $H$ a non-compact, Lie subgroup satisfying (2) with $G$ acting effectively on $G / H$. Such an $H$ will not satisfy (3). 
As a final observation we describe a way to construct new examples of pairs $(G, H), H$ satisfying (1), $H$ non-compact.

1.8. LeMMA. Let $(G, H)$ be a pair of a connected Lie group $G$ and a closed subgroup $H$ so that (1) holds. Let $(\sigma, \mathbf{V})$ be a finite dimensional representation of $G$ such that $H$ acts unitarily. Set $G_{\sigma}=\mathrm{V} \rtimes_{\sigma} G$. Assume that $\operatorname{ker}(\sigma) \cap \operatorname{ker}($ action of $G$ on $G / H)=\{e\}$. Then $G_{\sigma}$ acts effectively on $G_{\sigma} / H$ and $H$ satisfies (1). Furthermore, if $\operatorname{ker}(\sigma) \cap Z(G)=\{e\}, G$ acts without non-zero fixed vectors and $H$ is not compact, then (2) will not be satisfied.

Proof. Clearly $H$ is closed in $G_{\sigma}$ and $\operatorname{Ad}_{G_{o}}(H) \subset O(\mathbf{V}) \times \operatorname{Ad}_{G}(H)$, hence (1) holds. On the other hand

$$
\begin{aligned}
& \left(0, g_{1}\right) \cdot(v, g) \cdot\left(0, g_{1}^{-1}\right)=\left(\sigma\left(g_{1}\right) v, g_{1} g g_{1}^{-1}\right), \\
& \left(v_{1}, 1\right) \cdot(v, g) \cdot\left(-v_{1}, 1\right)=\left(v+v_{1}-\sigma(g) v_{1}, g\right) .
\end{aligned}
$$

Thus

$$
\bigcap_{x \in G_{\sigma}} x H x^{-1}=\left(\bigcap_{x \in G} x H x^{-1}\right) \cap \operatorname{ker} \sigma=\{e\},
$$

by the assumption. Hence the action is effective. On the other hand, by (2.5)

$$
Z\left(G_{\sigma}\right)=\left\{\left(g_{0}, v_{0}\right) ; g_{0} \in Z(G) \cap \operatorname{ker}(\sigma), \sigma(g) v_{0}=v_{0} \text { for any } g \in G\right\}
$$

and under the assumptions $Z\left(G_{\sigma}\right)=\{e\}$, hence the second assertion is clear.

1.9. Remarks. (a) We note that Example 1.6 is a particular instance of the construction in the lemma with $(G, H)=\left(\mathbf{R}^{n}, \mathbf{R}\right)$. In this case, the representation of $\mathbf{R}^{n}$ in $\mathbf{C}^{n+1}$ is faithful and unitary and the action of $G$ on $G / H$ is far from being effective but the action of $G_{\sigma}$ on $G_{\sigma} / H$ is so. This procedure could naturally be iterated by taking a finite dimensional representation $(\tau, \mathbf{W})$ of $G_{\sigma}$, unitary on $H$, thus getting a new pair $\left(\left(G_{\sigma}\right)_{\tau}, H\right)$.

(b) As another illustration of the lemma, let $G$ be semisimple and $H$ a closed non-compact subgroup such that (2) holds. Let $(\sigma, \mathbf{V})$ be a non-trivial, finite dimensional, irreducible representation of $G$. We have that $Z\left(G_{\sigma}\right)=\{(0, z) \mid z \in Z \cap \operatorname{ker} \sigma\}$. Hence

$$
p_{\sigma}\left(\overline{Z\left(G_{\sigma}\right) H}\right)=\{0\} \times p(\overline{(Z \cap \operatorname{ker} \sigma) H})
$$


is compact, where $p_{\sigma}: G_{\sigma} \rightarrow G_{\sigma} / Z\left(G_{\sigma}\right)$ is the canonical projection. Hence (2) holds and if, for instance, $G$ acts effectively or $\sigma$ is faithful the action of $G_{\sigma}$ is effective.

2. Non compact isotropy. In this section we consider the case when $G$ is semisimple. As observed in $\S 1$, for $H$ a closed subgroup of $G$ Conditions (1) and (2) are equivalent. The first result we prove is a characterization of connected non-compact subgroups $H$ satisfying (2). We also give a way to compute $\bar{G}_{L}$ explicitly, in the light of Proposition 1.2 and a classical result (see Theorem A.1 in the appendix). We also construct families of examples where $\operatorname{dim} \bar{G}_{L}-\operatorname{dim} G_{L}$ is the maximum possible.

Let then $G$ be a connected semisimple Lie group. If $G=K \cdot \exp (\mathfrak{p})$ is a Cartan decomposition, $K$ is compact if and only if $Z$, the center of $G$, is finite. Denote by $\mathfrak{f}$ the Lie algebra of $K$ and let $\mathfrak{f}^{\prime}=[\mathfrak{f}, \mathfrak{f}]$. Then $\mathfrak{f}=z_{\mathfrak{f}} \oplus \mathfrak{f}^{\prime}, z_{\mathfrak{f}}$ the center of $\mathfrak{f}$. Denote by $Z_{K}$ and $K^{\prime}$, respectively, the connected Lie subgroups of $K$ corresponding to $z_{\mathfrak{f}}$ and $\mathfrak{f}^{\prime}$. Then $K^{\prime}$ is compact and if furthermore, $G$ is simply connected, $K$ is isomorphic to the direct product of $Z_{K}$ and $K^{\prime}$. In this case $Z_{K}$ is a vector group.

2.1. Proposition. Let $G$ be a simply connected semisimple Lie group and $H$ a closed connected subgroup such that (2) is satisfied. Then there is a Cartan decomposition $G=K \exp (p)$ such that

$$
H=\exp (\mathbf{V}) \cdot\left(H \cap K^{\prime}\right)
$$

where $V$ is a vector subspace of the center of $\mathfrak{h}$, $\operatorname{exp:~} \mathbf{V} \rightarrow \exp \mathbf{V}$ is an isomorphism onto a closed subgroup and $\exp (\mathbf{V}) \cap\left(H \cap K^{\prime}\right)=1$. Furthermore $\overline{Z H}$ can be explicitly computed.

Proof. Let $p: G \rightarrow G / Z$ be the canonical projection. Since $p(\overline{Z H})$ $=\overline{p(H)}$ is compact, by a theorem of Iwasawa, there is a maximal compact subgroup $K_{0}$ of $G / Z$ such that $p(\overline{Z H}) \subset K_{0}$. Thus $\overline{Z H} \subset K=$ $p^{-1}\left(K_{0}\right)$. Let $K=Z_{K} \cdot K^{\prime}$ as above. If $Z_{k}=\{e\}$, then $K$ is compact, hence $H$, and (2.1) is clear. We thus assume from now on that $z_{\mathfrak{\xi}} \neq 0$. Now, $\mathfrak{h}$ admits an $\operatorname{Ad}(H)$-invariant inner product, hence $\mathfrak{h}=z_{0} \oplus \mathfrak{h}^{\prime}$ where $z_{0}$ is the center of $\mathfrak{h}$ and $\mathfrak{h}^{\prime}=[\mathfrak{h}, \mathfrak{h}]$. Let $H^{\prime}$ (resp. $Z_{H}$ ) be the connected Lie subgroup of $H$ corresponding to $\mathfrak{h}^{\prime}$ (resp. $z_{0}$ ). Then $H^{\prime}$ is compact and $H^{\prime} \subset K^{\prime}$. Write $z_{0}=\mathbf{V} \oplus z^{\prime}$ with $z^{\prime}=z_{0} \cap \mathfrak{f}^{\prime}$ and $\mathbf{V}$ a vector subspace of $z_{0}$. Fix $\left\{\left(U_{l}, Y_{l}\right): U_{l} \in z_{\mathfrak{f}}, Y_{l} \in \mathfrak{f}^{\prime}, 1 \leq i \leq m\right\}$ a basis of $\mathbf{V}$. Then the set $\left\{U_{i}: 1 \leq i \leq m\right\}$ is linearly independent since $\mathbf{V} \cap \mathfrak{f}^{\prime}=$ $\{0\}$. Also $\left[Y_{l}, Y_{j}\right]=0$, for all $i, j$. Assume now that

$$
\exp \left(\sum_{1}^{m} t_{l}\left(U_{i}, Y_{i}\right)\right)=\exp \left(\sum_{1}^{m} t_{i}\left(U_{l}, 0\right)\right) \exp \left(\sum t_{l}\left(0, Y_{i}\right)\right) \in K^{\prime} \text {. }
$$


Since $\exp \left(\sum_{1}^{m} t_{i} U_{i}\right)=1$, then, $t_{i}=0$ for $1 \leq i \leq m$. Hence, $\left.\exp \right|_{\mathbf{v}}$ is one-to-one and $\exp (\mathbf{V}) \cap K^{\prime}=\{1\}$. This implies that $Z_{H} \cap K^{\prime}=\exp \left(z^{\prime}\right)$, that is, $Z_{H}=\exp \mathrm{V} \cdot\left(Z_{H} \cap K^{\prime}\right)$.

Let now $v_{n} \in \mathbf{V}$ be so that $\exp \left(v_{n}\right) \rightarrow u \in G$. Then $u \in Z_{H}$, hence $u=\exp (v) u^{\prime}, u^{\prime} \in Z_{H} \cap K^{\prime}$. Write

$$
v_{n} v^{-1}=\sum_{1}^{m} t_{i, n}\left(U_{i}, Y_{i}\right) \text {. }
$$

Since $\exp \left(v_{n} v^{-1}\right) \rightarrow u^{\prime} \in K^{\prime}$ it follows that $\exp \left(\sum_{1}^{m} t_{i, n} U_{i}\right) \rightarrow 1$ in $Z_{K}$. Hence $t_{i, n} \rightarrow 0$, for $1 \leq i \leq m$ (again we use that $U_{1}, \ldots, U_{m}$ are linearly independent). Therefore $u^{\prime}=1$ and $\exp (\mathbf{V})$ is closed in $G$. This completes the proof of the first assertion. We now describe how to compute $\overline{Z H}$.

Fix $Z^{\prime} \subset Z \cap Z_{K}$, a subgroup such that $\left|Z / Z^{\prime}\right|<\infty$ and let $Z^{\prime \prime}$ be a system of representatives of $Z / Z^{\prime}$. Then

$$
\overline{Z H}=\overline{Z^{\prime} H} \cdot Z^{\prime \prime}=\overline{Z^{\prime} \cdot \exp \mathbf{V}} \cdot H \cap K^{\prime} \cdot Z^{\prime \prime} .
$$

Let $\left\{\left(U_{i}, Y_{i}\right) \mid 1 \leq i \leq m\right\}$ be as above. Also let $\mathfrak{g}=\sum_{1}^{m} \mathbf{R} Y_{i}, T^{\prime}$ $=\overline{\exp (\mathfrak{I})}$ and let $\mathrm{t}^{\prime}$ be the Lie algebra of $T^{\prime}$. Choose $\left\{X_{j}: 1 \leq j \leq d\right\}$ a basis of $\mathrm{t}^{\prime}$ so that $\sum \mathbf{Z} X_{j}=\operatorname{ker}\left(\left.\exp \right|_{\mathrm{t}^{\prime}}\right)$ and $\left\{V_{i}: 1 \leq i \leq c\right\}$ a basis of $z_{\mathfrak{f}}$ such that $Z^{\prime}=\exp \left(\sum \mathbf{Z} V_{i}\right)$.

Thus

$$
\overline{Z^{\prime} \exp (\mathbf{V})}=\exp \overline{\left(\sum_{1}^{c} \mathbf{Z} V_{i}+\sum_{1}^{d} \mathbf{Z} X_{j}+\mathbf{V}\right)}
$$

and it then follows from (2.2) and (2.3) that $\overline{Z H}$ can be computed (at least in principle) by means of Theorem A.1 in the appendix.

2.2. REMARK. Assume that $G$ is semisimple and the hypotheses of Proposition 1.3 hold. Then, given any $G$-invariant riemannian metric on $G / H$.

$$
\operatorname{dim} \bar{G}_{L} \leq \operatorname{dim} G+\operatorname{rank}(K)-\operatorname{rank}(H)
$$

where $G=K \cdot \exp (\mathfrak{p})$ is a Cartan decomposition of $G$. In fact, since $H$ satisfies (2), $\bar{G}_{L}$ is given by Proposition 1.3, hence

$$
\operatorname{dim} \bar{G}_{L}=\operatorname{dim} G+\operatorname{dim} \overline{Z H} / H .
$$

Now $\overline{Z H} \subset K=p^{-1}\left(K_{0}\right), K_{0}$ a maximal compact subgroup of $G / Z$, $p(\overline{Z H}) \subset K_{0}$. Let $\overline{\mathfrak{h}}$ denote the Lie algebra of $\overline{Z H}$. It is clear that $\mathfrak{h}$ is an ideal of $\overline{\mathfrak{h}}$ and there is an abelian ideal $\mathfrak{a}$ of $\overline{\mathfrak{h}}$ with $\overline{\mathfrak{h}}=\mathfrak{a} \oplus \mathfrak{h} \subset \mathfrak{f}$. Hence $\operatorname{dim} \bar{G}_{L}=\operatorname{dim} G+\operatorname{dim} \mathfrak{a} \leq \operatorname{dim} G+\operatorname{rank}(K)-\operatorname{rank}(H)$ as asserted. 
We next provide a family of examples where $\operatorname{dim} \bar{G}_{L}-\operatorname{dim} G_{L}$ is the maximum possible. This will show that the bound in (2.4) is sharp.

2.2. Proposition. Let $G$ be a simply connected semisimple Lie group such that the center of each simple factor is infinite. Then there exists a discrete subgroup $H \simeq \mathbf{Z}$ such that $\overline{Z H}=T$, a compact Cartan subgroup, and $G$ acts effectively on $G / H$. Furthermore if $G$ is not locally isomorphic to $\mathrm{SL}(2, R)$, there exists a closed subgroup $H^{\prime} \simeq \mathbf{R}$ with $\overline{Z H^{\prime}}=T$ and $G$ acting effectively on $G / H^{\prime}$. Then for any $G$-invariant riemannian metric on $G / H\left(\operatorname{resp} . G / H^{\prime}\right)$

$$
\operatorname{dim} \bar{G}_{L}=\operatorname{dim} G+\operatorname{rank}(K) \quad\left(\operatorname{resp} \cdot \operatorname{dim} \bar{G}_{L}=\operatorname{dim} G+\operatorname{rank}(K)-1\right) .
$$

Furthermore $\bar{G}_{L}=I(G / H)_{0}\left(\right.$ resp. $\left.I\left(G / H^{\prime}\right)_{0}\right)$.

Proof. Let $T$ be a Cartan subgroup, $T \subset K$ and let $\mathrm{t}$ denote the Lie algebra of $T$. Let $p: G \rightarrow G / Z$ be the canonical projection. Then $T / Z$ is a torus. Fix $X$ in $t$ so that, if $t=\exp (X), t Z$ is a generator of $T / Z$ (i.e. the powers of $t Z$ form a dense subgroup of $T / Z$ ). Let $H$ be the group generated by $t$ and let $H^{\prime}$ be the one-parameter group generated by $X$. Now $X=X_{1}+X_{2}, X_{1} \in z_{\mathfrak{k}}, X_{2} \in \mathfrak{f}^{\prime}$ and $X_{1} \neq 0$, since $t Z$ is a generator. This implies that both $H$ and $H^{\prime}$ are closed subgroups.

On the other hand $G$ acts effectively on $G / H$ since $\bigcap_{x \in G} x^{-1} T x=Z$ and by the choice of $t$ it is clear that $H \cap Z=\{e\}$. Similarly, if $H^{\prime} \cap Z=\{e\}$, the action on $G / H^{\prime}$ is effective. Otherwise $p\left(H^{\prime}\right)$ is a circle and then $p\left(H^{\prime}\right)=T$. Therefore $G$ is isomorphic to the universal covering group of $\operatorname{SL}(2, \mathbf{R})$, against our assumption. Thus, by (2.4) $\operatorname{dim} \bar{G}_{L}$ is as asserted, since, if $S=H$ or $H^{\prime}$, by Proposition $1.3 \bar{G}_{L}=G_{L} \cdot(\overline{Z S})_{R}$ $=G_{L} \cdot T_{R}$. Finally, by a result of C. Gordon ([G], Theorem 4.1)

$$
I(M)_{0}=G_{L} \cdot\left(U_{0}\right)_{R}
$$

where, as usual, $U=\left\{x \in N(S): R_{x}\right.$ is an isometry $\}$. Now

$$
T \subseteq U_{0} \subset N(S)_{0} \subset N(T)_{0}=T .
$$

Thus the last assertion is clear. This concludes the proof.

2.3. REMARK. It is easy to modify the example in (3.10) [DMW] to show that any connected semisimple Lie group with infinite center $G$ admits a closed, non-compact, discrete subgroup $H$ satisfying (2) and $G$ acting effectively on $G / H$. 
APPENDIX. In this appendix we list some classical facts on the subgroups of $\mathbf{R}^{n}$. We refer to [B], Ch. VII for a detailed treatment. If $x$, $y \in \mathbf{R}^{n}$ denote by $x \cdot y$ the canonical inner product. Given $G \subset \mathbf{R}^{n}$, a subgroup, set $G^{*}=\left\{g^{\prime} \in \mathbf{R}^{n} \mid g^{\prime} \cdot g \in \mathbf{Z}\right.$, for all $\left.g \in G\right\}$.

A.1. Theorem (see [B], p. 74). Let $G \subset \mathbf{R}^{n}$ be a subgroup. Then $\bar{G}=G^{* *}$. In particular $G$ is dense if and only if $G^{*}=\{0\}$.

A.2. Corollary. Fix $v=\left(a_{1}, \ldots, a_{n}\right) \in \mathbf{R}^{n}$ and let $\left\{e_{j}\right\}_{1}^{n}$ be the canonical basis. Let $G_{1}=\mathbf{Z} v+\sum_{1}^{n} \mathbf{Z} e_{j}$ (resp. $G_{2}=\mathbf{R} v+\sum_{1}^{n} \mathbf{Z} e_{j}$ ). Then $G_{1}$ (resp. $G_{2}$ ) is dense in $\mathbf{R}^{n}$ if and only if $1, a_{1}, \ldots, a_{n}$ are $\mathbf{Q}$-linearly independent (resp. $a_{1}, \ldots, a_{n}$ are $\mathbf{Q}$-linearly independent).

A.3. Lemma. If $1 \leq k \leq n$, let $\phi: \mathbf{R}^{n} \rightarrow \mathbf{R}^{k} \times T^{n-k}$ be the canonical map, $\phi\left(t_{1}, \ldots, t_{k}, t_{k+1}, \ldots, t_{n}\right)=\left(t_{1}, \ldots, t_{k}, e^{2 \pi i t_{k+1}}, \ldots, e^{2 \pi i t_{n}}\right)$. If $G \subset \mathbf{R}^{n}$ is a subgroup, then $\overline{\phi(G)}=\phi\left(\overline{G+\sum_{k+1}^{n} \mathbf{Z} e_{j}}\right)$.

A.4. LemMa. There exists an $n$-dimensional subspace $\mathbf{V}$ of $R^{n+1}$ and $v \in \mathbf{V}$ such that $\mathbf{V} \cap \sum_{1}^{n+1} \mathbf{Z} e_{j}=\{0\}$ and $\mathbf{Z} v+\sum_{1}^{n+1} \mathbf{Z} e_{j}$ is dense in $\mathbf{R}^{n+1}$.

Proof. The lemma follows in a standard fashion from Theorem A.1. The proof will thus be omitted.

\section{REFERENCES}

[B] N. Bourbaki, General Topology, Part 2, Hermann 1966.

[ChE] J. Cheeger and D. Ebin, Comparison Theorems in Riemannian Geometry, North Holland Publishing Company, Amsterdam-Oxford.

[G] C. Gordon, Riemannian isometry groups containing transitive reductive subgroups, Math. Ann., 248 (1980), 185-192.

[H] S. Helgason, Differential Geometry, Lie Groups, and Symmetric Spaces, Academic Press, 1978.

[DMW] I. Dotti Miatello, R. J. Miatello and J. A. Wolf, Bounded isometries and homogeneous quotient manifolds, Geometriae Dedicata, to appear.

Recieved August 5, 1986 and in revised form October 28, 1986. Partially supported by Conicet, Argentina.

Facultad de Matemática, Astronomía y Física (FAMAF)

UNIVERSIDAD NACIONAL DE CORDOBa

Avdas. Valparatso y R. Martínez

5000 - CORDOBA - ARGENTINA 


\section{PACIFIC JOURNAL OF MATHEMATICS EDITORS}

\author{
V. S. VARADARAJAN \\ (Managing Editor) \\ University of California \\ Los Angeles, CA 90024 \\ Herbert Clemens \\ University of Utah \\ Salt Lake City, UT 84112 \\ R. FINN \\ Stanford University \\ Stanford, CA 94305
}

ROBION KIRBY

University of California

Berkeley, CA 94720

C. C. MOORE

University of California

Berkeley, CA 94720

HAROLD STARK

University of California, San Diego

La Jolla, CA 92093

\section{ASSOCIATE EDITORS}
R. ARENS
E. F. BECKENBACH
B. H. NEUMANN
F. WOLF
K. YOSHIDA (1906-1982)

\section{SUPPORTING INSTITUTIONS}
UNIVERSITY OF ARIZONA
UNIVERSITY OF OREGON
UNIVERSITY OF BRITISH COLUMBIA UNIVERSITY OF SOUTHERN CALIFORNIA
CALIFORNIA INSTITUTE OF TECHNOLOGY
UNIVERSITY OF CALIFORNIA
STANFORD UNIVERSITY
MONTANA STATE UNIVERSITY
UNIVERSITY OF HAWAII
UNIVERSITY OF NEVADA, RENO
UNIVERSITY OF TOKYO
NEW MEXICO STATE UNIVERSITY
UNIVERSITY OF UTAH
OREGON STATE UNIVERSITY
WASHINGTON STATE UNIVERSITY UNIVERSITY OF WASHINGTON 


\section{Pacific Journal of Mathematics}

\section{Vol. 131, No. $1 \quad$ November, 1988}

Tomek Bartoszynski, On covering of real line by null sets $\ldots \ldots \ldots \ldots \ldots 1$

Allen Davis Bell and Kenneth R. Goodearl, Uniform rank over differential operator rings and Poincaré-Birkhoff-Witt extensions $\ldots \ldots \ldots \ldots \ldots 13$

Brian Boe, Thomas Jones Enright and Brad Shelton, Determination of the intertwining operators for holomorphically induced representations of Hermitian symmetric pairs

Robert F. Brown, Topological identification of multiple solutions to

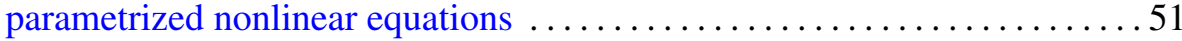

Marc R. M. Coppens, Weierstrass points with two prescribed nongaps ......71

Peter Larkin Duren and M. Schiffer, Grunsky inequalities for univalent functions with prescribed Hayman index .................... 105

Robert Greene and Hung-Hsi Wu, Lipschitz convergence of Riemannian manifolds

Kathryn E. Hare, Arithmetic properties of thin sets

Neal I. Koblitz, Primality of the number of points on an elliptic curve over a

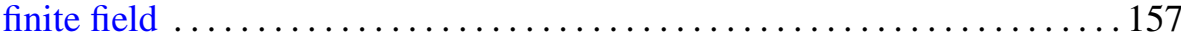

Isabel Dotti de Miatello and Roberto Jorge Miatello, Transitive isometry groups with noncompact isotropy

Raymond A Ryan, Weakly compact holomorphic mappings on Banach

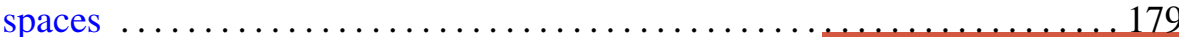

Tudor Zamfirescu, Curvature properties of typical convex surfaces 